\title{
Evaluation of protein extraction protocols for 2DE in marine ecotoxicoproteomics
}

\author{
Huifeng $\mathrm{Wu}^{1}$, Chenglong $\mathrm{Ji}^{1,2}$, Lei Wei ${ }^{1,2}$ and Jianmin Zhao ${ }^{1}$ \\ ${ }^{1}$ Key Laboratory of Coastal Zone Environmental Processes, Yantai Institute of Coastal Zone Research (YIC), \\ Chinese Academy of Sciences (CAS); Shandong Provincial Key Laboratory of Coastal Zone Environmental \\ Processes, YICCAS, Yantai, P. R. China \\ ${ }^{2}$ The Graduate School of Chinese Academy of Sciences, Beijing, P. R. China
}

In ecotoxicoproteomics, an accurate and reproducible extraction of proteins is a critical step for 2DE analysis and further protein identification using MS. The criteria for the assessment of protein extraction quality include protein yield, protein spots resolved in a 2DE gel, matched protein spots in replicate gels, reproducibility, and compatibility with MS. In this work, we evaluated three protein extraction systems, straightforward lysis buffer, trichloroacetic acidacetone, and TRIzol reagent with some modifications, for the protein extraction from three animal species including mussel Mytilus galloprovincialis, flounder Paralichthys olivaceus, and polychaete Nereis diversicolor used in marine ecotoxicology. Our results indicated that these methods could extract significantly different protein profiles. The method using TRIzol reagent resulted in the most matched protein spots resolved in four replicate 2DE gels and highest reproducibilities for the gill of $M$. galloprovincialis and liver of $P$. olivaceus. However, a modified trichloroacetic acid-acetone solvent system was best for the whole soft tissue of $N$. diversicolor. This work provides the fundamental information of the extraction quality of protein extraction protocols from different marine animals, which may facilitate the selection of a suitable protein extraction protocol for ecotoxicoproteomics.

\section{Keywords:}

2DE / Animal proteomics / Ecotoxicoproteomics / Protein extraction / Reproducibility

Additional supporting information may be found in the online version of this article at the publisher's web-site
Received: September 12, 2012

Revised: August 25, 2013

Accepted: August 28, 2013
Proteomics is the large-scale study of proteins encoded by a genome in an organism [1]. Since comparative proteomics can directly compare the protein expression levels in an organism, it can provide an overall view of toxicological mechanisms of contaminants and has, therefore, been widely used in ecotoxicology resulting in the emergence of "ecotoxicoproteomics” [2]. Proteomics usually involves extraction, separa-

Correspondence: Dr. Huifeng Wu, Yantai Institute of Coastal Zone Research, Chinese Academy of Sciences, Yantai 264003, P. R. China

E-mail: hfwu@yic.ac.cn

Fax: $+86-535-2109000$

Abbreviations: PC, principal component; PCA, principal component analysis; Phe, phenol; TCA, trichloroacetic acid tion, and identification of proteins in biological samples to produce unique protein profiles. Comparison of such protein profiles can then be used to identify changes in protein expression levels combined with MS and software databases [2]. 2DE was established in the 1970's and has been rapidly developed for proteomic analysis [3]. In recent years, although some alternative gel-free techniques, such as multidimensional LC with stable isotopic labeling [4] and antibody arrays [5], have been developed, 2DE remains useful in resolving protein extracts from organisms due to its high resolution and low expense [6,7]. Obviously, an accurate and reproducible preparation of proteins is the most important step for 2DE analysis.

Several protein extraction systems, such as straightforward lysis buffer solution, trichloroacetic acid (TCA)-based and phenol- based extraction systems, have been applied in marine ecotoxicoproteomics for the extraction of proteins from sentinel animals, although possible modifications are 


\section{Gill of M. galloprovincialis}
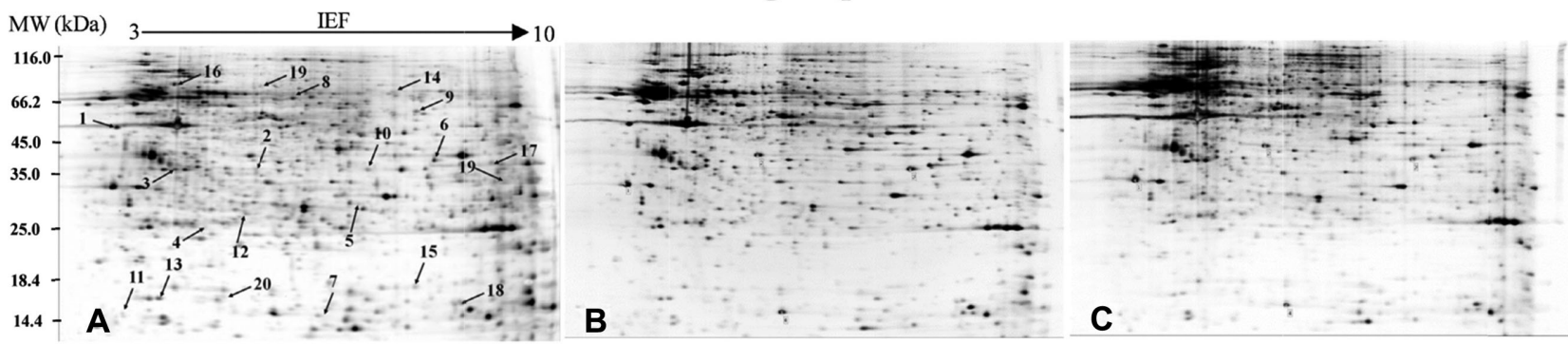

Liver of $P$. olivaceus
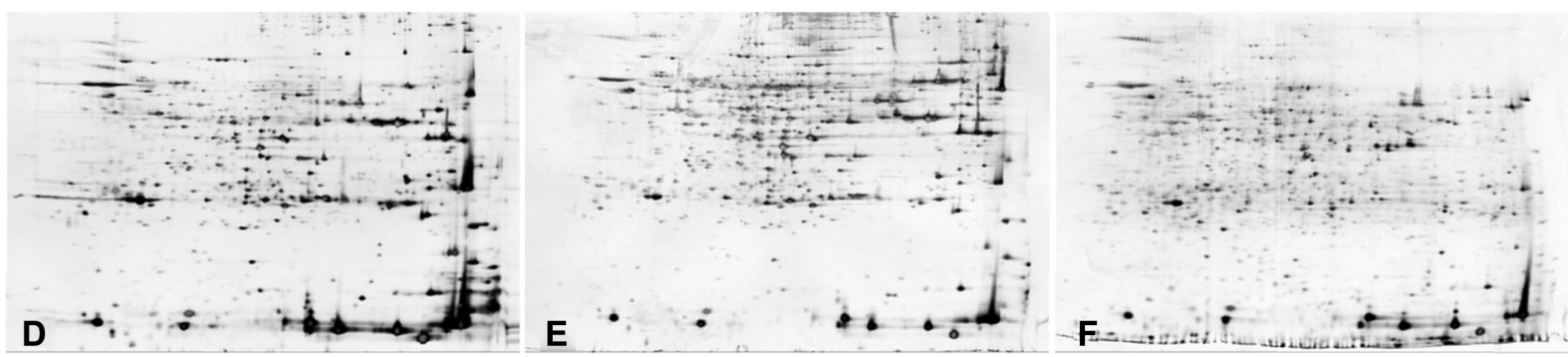

\section{Whole soft tissue of $N$. diversicolor}

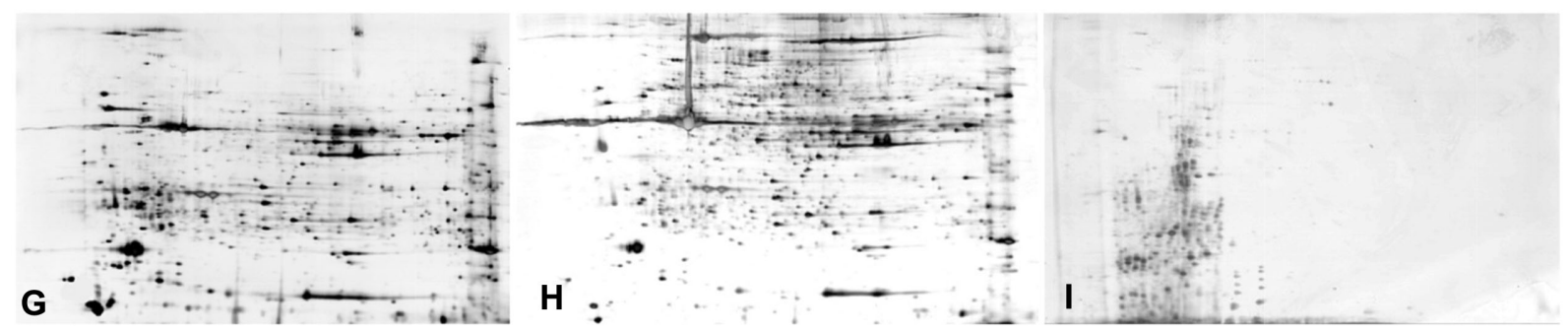

Figure 1. 2DE analysis of proteins from gill of $M$. galloprovincialis, liver of $P$. olivaceus, and whole soft tissue of $N$. diversicolor, extracted using the methods of TRIzol reagent ( $A, D$, and $G)$, TCA-acetone (B, E, and H), and straightforward lysis buffer (C, F, and I). Proteins were separated on a 3-10 linear $\mathrm{pH}$ gradient in the first dimension and visualized using silver staining. The numbered arrows indicate proteins that were identified by MS/MS and are listed in Supporting Information Table 1.

unavoidable [8-10]. The straightforward lysis buffer solutions (e.g. $7 \mathrm{M}$ urea, $2 \mathrm{M}$ thiourea, $4 \% \mathrm{~m} / \mathrm{v}$ CHAPS, $65 \mathrm{mM}$ DTT, and $0.2 \% \mathrm{w} / \mathrm{v}$ Bio-lyte buffer) with or without Tris and enzyme inhibitor are frequently used for protein extraction from animals because of its efficiency for the recovery of proteins with a high yield $[8,11]$. Since both TCA and acetone are efficient protein precipitation reagents, a combination of TCA and acetone (TCA-acetone) is widely applied for protein extraction with different modifications [12]. For example, in earthworm Eisenia fetida, the TCA-acetone extraction method resulted in good distinction and a large number of protein spots in 2DE gels compared with other extraction protocols [1]. Jiang et al. reported that an addition of $40 \mathrm{mM}$ Tris before precipitation using TCA-acetone could make a good extraction of proteins from Chinese shrimp Fenneropenaeus chinensis [9]. As it is known, TRIzol reagent is used for isolation of RNA from biological samples. However, after RNA extraction, this extraction protocol can be used for protein precipitation from its phenol supernatant [11]. Apparently, a concurrent extraction of RNA and protein is important when the correlation between gene and protein expressions needs to be analyzed.

In marine ecotoxicology, mussel Mytilus galloprovincialis [13], flounder Paralichthys olivaceus [14], and polychaete Nereis diversicolor [15] are often selected as experimental animals for elucidating toxicological effects and mechanisms of contaminants. These species have also been successfully used in marine ecotoxicoproteomics $[8,16,17]$. Although different protein extraction protocols, such as straightforward lysis buffer solution and TCA-acetone, were technically usable for protein extraction from marine animals, an evaluation of the quality of these protein extraction protocols is necessary for 2DE. In this work, three protein extraction systems including straightforward lysis buffer solution, TCA-acetone, and TRIzol reagent were optimized and evaluated for protein extraction from M. galloprovincialis, P. olivaceus, and N. diversicolor. The gill tissue of M. galloprovincialis, liver of P. olivaceus, 
and whole soft tissue of $N$. diversicolor were sampled from single individuals of these species. However, the optimization for straightforward lysis buffer did not result in good extraction of protein, this method was not fully evaluated for $N$. diversicolor. In ecotoxicoproteomics, minor proteomic differences between control- and contaminant-exposed groups are often meaningful. However, these differences could be generated from both technical and biological sources. Therefore, it is necessary to minimize technical differences to obtain maximum biological differences induced by contaminants. In this work, we aimed to assess the quality of these protein extraction protocols in terms of protein yield, number of protein spots separated in 2DE gels, and reproducibility.

Before the determination of the final formula and procedures for each solvent system, we primarily assessed the 2DE gel quality of each solvent system with different modifications described in the Supporting Information. The number of protein spots, resolution of protein spots, and gel background were assessed for the determination of final formula and extraction procedures for each extraction system. Eventually, the extraction systems of TCA-acetone with a prior addition of Tris and the straightforward lysis buffer with an addition of Tris and enzyme inhibitors resulted in the best quality of 2DE gels for all study samples. Then these two modified solvent systems of TCA-acetone and straightforward lysis buffer were used for the further quantitative evaluation for protein extractions together with TRIzol reagent.

The marine mussel genus Mytilus is one of the most frequently used sentinel organisms in marine ecotoxicology $[13,18]$. It was fully evaluated for the quality assessment of extraction systems. Figure $1 \mathrm{~A}-\mathrm{C}$ presents the silver-stained 2DE gels from the same sample of a gill tissue extracted by TRIzol, modified straightforward lysis buffer, and TCAacetone. Clearly, all the three extraction methods resulted in good separations of protein spots in corresponding four technical replicate 2DE gels. Further image analysis of 2DE gels indicated that TRIzol extraction presented the largest number of protein spots $(1065 \pm 95, n=4)$ than TCA-acetone $(986 \pm 79, n=4)$ and straightforward lysis buffer (920 \pm $75, n=4)$. Although a maximum number of protein spots in a $2 \mathrm{DE}$ gel are always a primary request in proteomics, a maximum number of matched spots from replicate samples are more crucial for an extraction protocol, which can potentially present more proteomic differences. After detailed image analysis of 2DE gels, a total of 759 matched spots were found in four technical replicate gels from TRIzol reagent extracted samples, together with 553 and 505 matched spots in technical replicate gels from TCA-acetone and straightforward lysis buffer protocols, respectively. It showed that TRIzol reagent gave a highest number of matched proteins than the other two extraction methods. To further compare the reproducibility of each extraction method, PCA was applied to the vol (\%) values of the commonly matched protein spots (329) in all the 12 gels for the generation of principal component (PC) scores. PCA resulted in significant $(p<0.01)$ separations between the groups of three protein
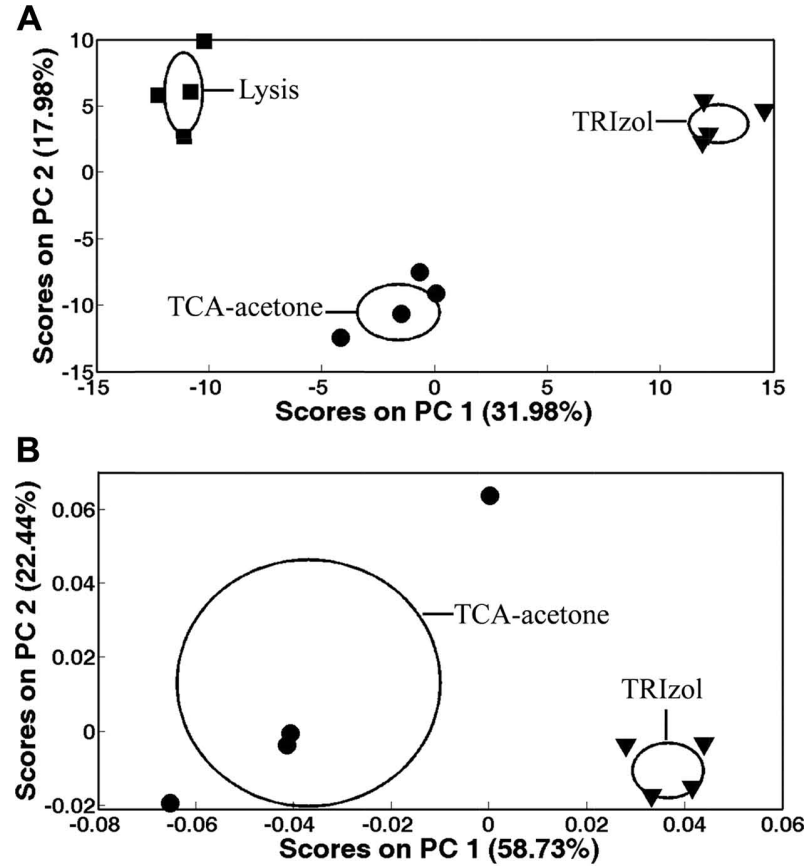

C

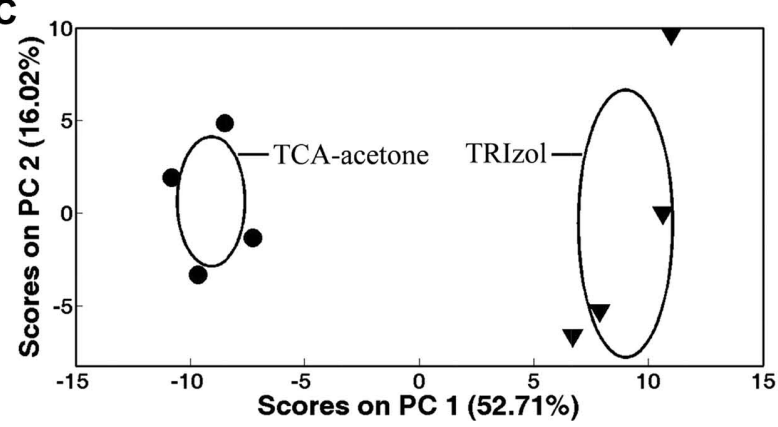

Figure 2. PCA scores plots from analysis of the percentage volume values (\% vol) from the commonly matched protein spots in all 2DE gels ( $n=4$ for each extraction method) for gill of M. galloprovincialis $(\mathrm{A})$, liver of $P$. olivaceus $(\mathrm{B})$ and whole soft tissue of $N$. diversicolor $(C)$. Due to the low number of matched protein spots across the four technical replicate gels, the method using straightforward lysis buffer was excluded from corresponding PCA analysis for the samples from either liver of $P$. olivaceus or whole soft tissue of $N$. diversicolor. Ellipses represent mean \pm SD for each extraction method.

extraction methods, implying that different extraction methods generated dissimilar proteomic profiles and thus a reproducible extraction method is necessary. The reproducibility of each protein extraction method was determined from PC scores (Fig. 2) by multiplying SD along PC1 by SD along PC2 for the replicates within each extraction method. To facilitate the comparison of reproducibilities of protein extraction methods, we normalized the reproducibility of TRIzol method tob 1 (Supporting Information Table 1). Although both TCA-acetone and straightforward lysis buffer resulted in higher extraction yields of proteins (Supporting Information Table 1), the extraction method with TRIzol reagent was the 

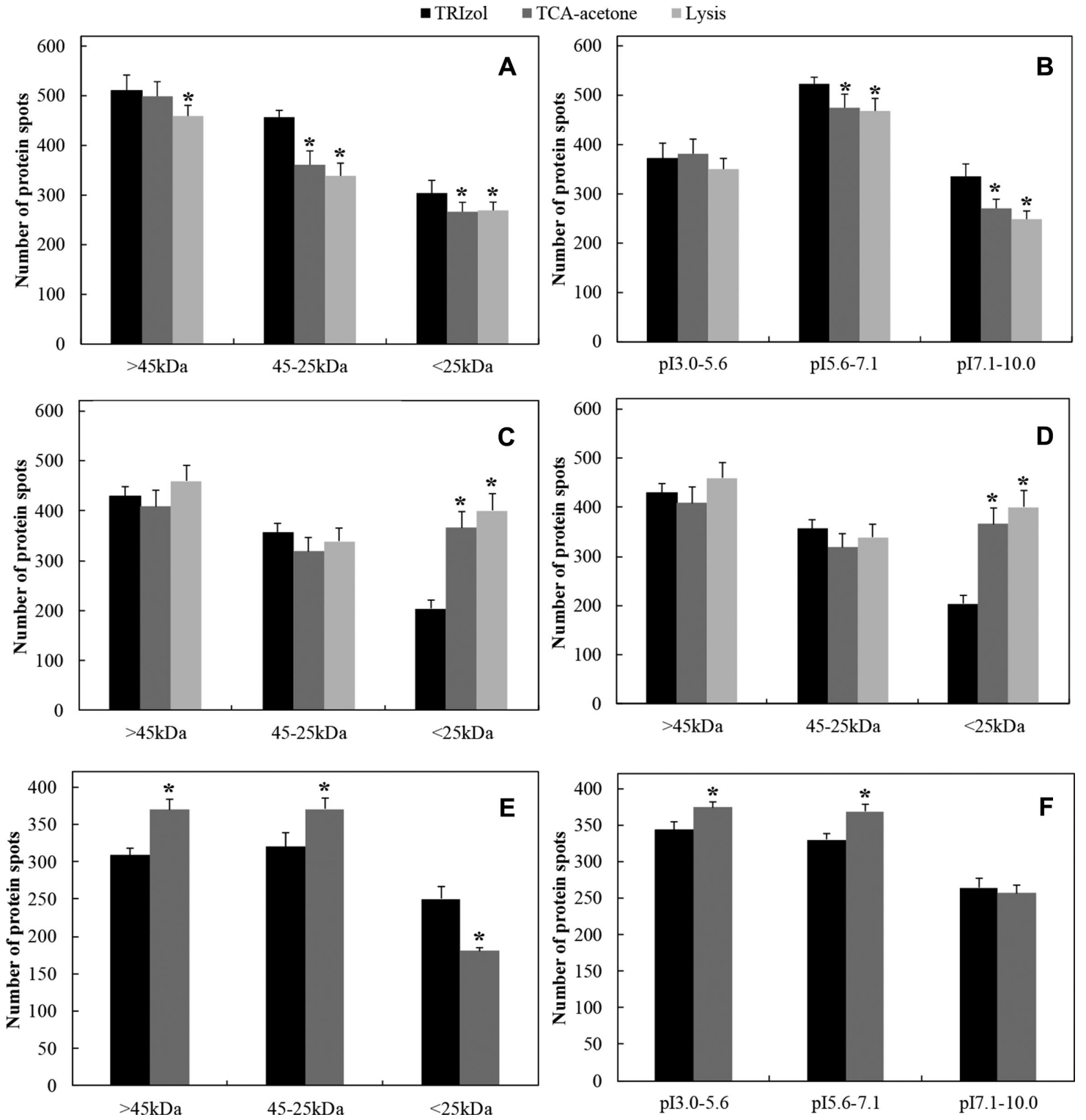

Figure 3. Number of protein spots from gill of $M$. galloprovincialis $(A+B)$, liver of $P$. olivaceus $(C+D)$ and whole soft tissue of $N$. diversicolor $(E+F)$ detected in $2 \mathrm{DE}$ gels using the extraction methods of TRlzol reagent, TCA-acetone, and straightforward lysis. Spots were classified into different ranges of molecular weight ( $>45,45-25$, and $<25 \mathrm{kDa}$ ) and $\mathrm{p} /(3.0-5.6,5.6-7.1$, and $7.1-10.0)$. Values are presented as the mean \pm SD of the numbers of spots from four technical replicates. Due to the low number of protein spots presented in 2DE gels, the method using straightforward lysis buffer was excluded from statistical analysis for the samples from whole soft tissue of $N$. diversicolor. Statistical differences $\left({ }^{*}, p<0.05\right)$ of protein spots between TRIzol reagent and TCA-acetone, and between TRIzol reagent and straightforward lysis were determined by one-way ANOVA with Tukey's test.

most reproducible method for protein extraction from the gill tissue of M. galloprovincialis (Fig. 3A and Supporting Information Table S1).

Flounder P. olivaceus is a nonsentinel organism, however, it has been often studied in toxicology [14]. In addition, a couple of proteomic studies have focused on the toxicological responses in $P$. olivaceus to cadmium exposure $[16,19]$. In this study, we used the liver of $P$. olivaceus for the evaluation of protein extraction methods since it is the main detoxification organ. Our results indicated that both straightforward lysis buffer solution and TCA-acetone extracted $>1100$ proteins from the same liver samples by analyzing corresponding 2DE gels (Supporting Information Table 1). However, the method of straightforward lysis buffer solution extracted fewer than half the number of matched proteins (285) of those (601) extracted by TRIzol, which indicated the limitation of straightforward lysis buffer solution for protein extraction from the liver of $P$. olivaceus. Therefore, the straightforward lysis buffer 
solution was not qualified for further comparisons. Based on the analysis of the four technical replicate 2DE gels, there were 601 and 406 matched protein spots from the proteins extracted by TRIzol reagent and TCA-acetone, respectively. Moreover, TRIzol reagent revealed a 19 times higher reproducibility than TCA-acetone. These results suggested that the protein extraction method using TRIzol reagent was better for protein extraction from the liver of $P$. olivaceus.

Like mussel $M$. galloprovincialis, the polychaete $N$. diversicolor has also been widely utilized as a sentinel animal in marine ecotoxicology $[15,20]$, and therefore was selected to evaluate the suitability of protein extraction methods. The whole fresh soft tissue of an individual $N$. diversicolor was processed for the assessment of protein extraction methods. As shown in Supporting Information Table 1, TRIzol reagent and TCA-acetone exhibited approximately 900 and 1000 proteins, respectively, from replicate samples. Surprisingly, straightforward lysis buffer solutions with different modifications extracted the lowest number of protein spots (340) resolved in silver-stained 2DE gels. However, Mok et al. (2009) found approximately 660 proteins in 2DE gels from another polychaete Pseudopolydora vexillosa using straightforward lysis buffer solution [21]. The species difference might be accounted for the differences in protein extraction. Further analysis indicated that TCA-acetone extracted more matched proteins (712) than TRIzol reagent (427) from the analysis of four technical replicate 2DE gels. In addition, TCA-acetone method showed higher reproducibility of protein extraction than TRIzol reagent (Supporting Information Table 1), which was completely different from the results of the samples of M. galloprovincialis and P. olivaceus.

A lysis buffer is usually preferable for protein recovery, and hence higher yield was obtained. However, it lacked proteins in median molecular weight range, which was observed in the gill of M. galloprovincialis as well (Fig. 3). The extraction quality of lysis buffer for the samples from M. galloprovincialis and P. olivaceus was lower than TRIzol reagent in terms of fewer matched protein spots in 2DE gels and lower reproducibilities. Moreover, the number of spots for TRIzol reagent at the 5.6-10.0 $\mathrm{pI}$ and $45-25 \mathrm{kDa}$ ranges was greater than other two methods (Fig. 3). TCA-acetone is a classical extraction system due to its effective precipitation of proteins and instant elimination of proteolytic enzymes. For the samples of $N$. diversicolor, however, TCA-acetone exhibited its better protein extraction quality than TRIzol, which implies that TRIzol reagent is not universally better than TCA-acetone. Since TRIzol method had two procedures to precipitate proteins using TRIzol and ethanol, while other two methods had one procedure to precipitate proteins, TRIzol resulted in relatively high protein loss (mainly those proteins with high abundances) and low protein yields (Supporting Information Table 1). However, it has been demonstrated that TRIzol can extract a wide repertoire of proteins of both high and low molecular weights [11]. Therefore, TRIzol could extract higher number of matched protein spots across technical replicate gels than other two methods. Besides the repro- ducibility, the compatibility of extracted proteins with MS is also crucial in ecotoxicoproteomics. In order to test the compatibility between silver-stained proteins and MS, 20 protein spots extracted by both TCA-acetone and TRIzol from M. galloprovincialis gill were excised from a silver-stained 2DE gel and analyzed by MS/MS. As a result, 15 proteins were successfully identified (Supporting Information Table 2). This confirmed the compatibility between silver-stained proteins and MS. Overall, the TRIzol reagent usually can produce acceptable protein resolution and reproducibility, and can be adopted for the gill of M. galloprovincialis and liver of $P$. olivaceus. It is preferable especially when the analysis of protein and RNA analysis in parallel is needed. A TCA-acetone-based extraction system may be used for $N$. diversicolor with a good reproducibility. This work provided the fundamental information of the quality of various protein extraction protocols from different marine animals. However, the selection of a suitable protein extraction protocol depends on the study objective.

This work was supported by NSFC (21237004), Natural Science Foundation of Shandong Province (Appl. No. 2013JQF01012) and Key Deployment Program of Chinese Academy of Sciences (KZZD-EW-14-03).

The authors have declared no conflict of interest.

\section{References}

[1] Anderson, N. L., Anderson, N. G., Proteome and proteomics: new technologies, new concepts, and new words. Electrophoresis 1998, 19, 1853-1861.

[2] Lemos, M. F. L., Soares, A. M. V. M., Correia, A. C., Esteves, A. C., Proteins in ecotoxicology-how, why and why not? Proteomics 2010, 10, 873-887.

[3] O'Farrell, P. H., High resolution two-dimensional electrophoresis of proteins. J. Biol. Chem. 1975, 250, 4007-4021.

[4] Roe, M. R., Griffin, T. J., Gel-free mass spectrometry-based high throughput proteomics: tools for studying biological response of proteins and proteomes. Proteomics 2006, 6, 4678-4687.

[5] Barry, R., Soloviev, M., Quantitative protein profiling using antibody arrays. Proteomics 2004, 4, 3717-3726.

[6] Choi, J.-W., Liu, H., Song, H., Park, J. H. Y., Yun, J. W., Plasma marker proteins associated with the progression of lung cancer in obese mice fed a high-fat diet. Proteomics 2012, 12, 1999-2013.

[7] Liu, H., Choi, J. -W., Yun, J. W., Gender differences in rat plasma proteome in response to high-fat diet. Proteomics 2012, 12, 269-283.

[8] Puertoa, M., Campos, A., Prietoa, A., Cameána, A. et al., Differential protein expression in two bivalve species; Mytilus galloprovincialis and Corbicula fluminea; exposed to Cylindrospermopsis raciborskii cells. Aquat. Toxicol. 2011, 101, 109-116. 
[9] Jiang, H., Li, F., Xie, Y., Huang, B. et al., Comparative proteomic profiles of the hepatopancreas in Fenneropenaeus chinensis response to hypoxic stress. Proteomics 2009, 9, 3353-3367.

[10] Saravanan, R. S., Rose, J. K. C., A critical evaluation of sample extraction techniques for enhanced proteomic analysis of recalcitrant plant tissues. Proteomics 2004, 4, 2522-2532.

[11] Likhite, N., Warawdekar, U. M., A unique method for isolation and solubilization of proteins after extraction of RNA from tumor tissue using Trizol. J. Biomol. Tech. 2011, 22, 37-44.

[12] Wang, X., Chang, L., Wang, G., Sun, Z. et al., Protein extraction from the earthworm Eisenia fetida for 2-DE. Proteomics 2010, 10, 1095-1099.

[13] Ciacci, C., Barmo, C., Gallo, G., Maisano, M. et al., Effects of sublethal, environmentally relevant concentrations of hexavalent chromium in the gills of Mytilus galloprovincialis. Aquat. Toxicol. 2012, 120-121, 109-118.

[14] Dong, X., Zhang, X., Qin, J., Zong, S., Acute ammonia toxicity and gill morphological changes of Japanese flounder Paralichthys olivaceus in normal versus supersaturated oxygen. Aquac. Res. 2012, 1-8.

[15] Li, L., Liu, X., You, L., Zhang, L. et al., Uptake pathways and subcellular fractionation of $\mathrm{Cd}$ in the polychaete Nereis diversicolor. Ecotoxicology 2012, 21, 104-110.
[16] Ling, X.-P., Zhu, J.-Y., Huang, L., Huang, H.-O., Proteomic changes in response to acute cadmium toxicity in gill tissue of Paralichthys olivaceus. Environ. Toxicol. Pharmacol. 2009, 27, 212-218.

[17] Ruffin, P., Demuynck, S., Hilbert, J. L., Dhainaut, A., Stress proteins in the polychaete annelid Nereis diversicolor induced by heat shock or cadmium exposure. Biochimie 1994, $76,423-427$.

[18] Li, A., Ma, J., Cao, J., McCarron, P., Toxins in mussels (Mytilus galloprovincialis) associated with diarrhetic shellfish poisoning episodes in China. Toxicon 2012, 60, 420-425.

[19] Zhu, J.-Y., Huang, H.-Q., Bao, X.-D., Lin, Q.-M., Cai, Z., Acute toxicity profile of cadmium revealed by proteomics in brain tissue of Paralichthys olivaceus: potential role of transferrin in cadmium toxicity. Aquat. Toxicol. 2006, 78, 127-135.

[20] Tankoua, O. F., Buffet, P. E., Amiard, J. C., Amiard-Triquet, C. et al., Intersite variations of a battery of biomarkers at different levels of biological organisation in the estuarine endobenthic worm Nereis diversicolor (Polychaeta, Nereididae). Aquat. Toxicol. 2012, 114-115, 96-103.

[21] Mok, F. S. Y., Thiyagarajan, V., Qian, P. -Y., Proteomic analysis during larval development and metamorphosis of the spionid polychaete Pseudopolydora vexillosa. Proteome Sci. 2009, 7, 44-54. 